\title{
Micro Soft Tissues Visualization Based on X-Ray Phase-Contrast Imaging
}

\author{
Lu Zhang and Shuqian Luo*
}

\author{
School of Biomedical Engineering, Capital Medical University, Beijing, China
}

\begin{abstract}
The current imaging methods have a limited ability to visualize microstructures of biological soft tissues. Small lesions cannot be detected at the early stage of the disease. Phase contrast imaging (PCI) is a novel non-invasive imaging technique that can provide high contrast images of soft tissues by the use of X-ray phase shift. It is a new choice in terms of non-invasively revealing soft tissue details. In this study, the lung and hepatic fibrosis models of mice and rats were used to investigate the ability of PCI in microstructures observation of soft tissues. Our results demonstrated that different liver fibrosis stages could be distinguished non-invasively by PCI. The three-dimensional morphology of a segment of blood vessel was constructed. Noteworthy, the blood clot inside the vessel was visualized in three dimensions which provided a precise description of vessel stenosis. Furthermore, the whole lung airways including the alveoli were obtained. We had specifically highlighted its use in the visualization and assessment of the alveoli. To our knowledge, this was the first time for non-invasive alveoli imaging using PCI. This finding may offer a new perspective on the diagnosis of respiratory disease. All the results confirmed that PCI will be a valuable tool in biological soft tissues imaging.
\end{abstract}

Keywords: Phase contrast imaging (PCI), diffraction enhanced imaging (DEI), in-line X-ray phase contrast imaging (IL$\mathrm{XPCI}$ ), hepatic fibrosis, microvessel, alveoli.

\section{INTRODUCTION}

The occurrence and development of a disease are usually accompanied by organ or tissue morphology changes [1-3]. Finding ways to evaluate these changes more accurately and efficiently becomes a key factor in the success of disease diagnosis and research. In the last decades, clinical imaging has been accelerated by the introduction of many new imaging technologies such as X-ray computed tomography (CT) and Magnetic resonance (MR) imaging. However, minor pathological changes, especially at the early stage as yet cannot be detected [4]. Since conventional CT relies on absorption as the contrast mechanism, which is not sensitive to soft tissues; MR imaging is limited by its poor spatial resolution and long acquisition time [5]. For instance, lung cancer is one of the most common fatal diseases worldwide. Early detection of the lung cancer is crucial for successful treatment and high survival rate. But in most cases, cancer has been detected in the terminal stage and missed the optimal time for cure [6]. High resolution non-invasive imaging technology will prove to be valuable tools under such circumstance.

Phase contrast imaging (PCI) is the imaging modality of choice for non-invasive and high resolution imaging of soft tissues. Conventional X-ray imaging is an attenuation-based technique. Unfortunately, when imaging soft tissues, the attenuation variations are not able to produce enough image contrast. Actually, when the X-ray propagates through an object, the phase modifies as well. The complex refractive index of the sample can be given by $n=1-\delta-i \beta$ [7]. The real part $\delta$ is related to the X-ray phase shift, while the imaginary

*Address correspondence to this author at the School of Biomedical Engineering, Capital Medical University, Beijing, China;

Tel: 86-10-83911549; Fax: 86-10-84719024; E-mail: sqluo@ieee.org part $\beta$ is responsible for $\mathrm{X}$-ray attenuation. For biological soft tissues, the phase shift is almost one thousand times greater than the absorption term for X-ray at $10-100 \mathrm{keV}$ [8]. Therefore, by the use of X-ray phase shift, phase contrast imaging can provide high contrast soft tissues images.

To date, several methods of PCI have been proposed including interferometry, diffraction enhanced imaging (DEI), in-line X-ray phase contrast imaging (IL-XPCI) and grating phase contrast imaging [7, 9-11]. Among all the main PCI methods, DEI has drawn considerable attention in biomedical field [12]. It utilizes an analyzer crystal with high angular sensitivity to measure the direction changes of the transmitted beam through the sample and then generates images of a sample's absorption and refraction properties. The method of in-line X-ray phase contrast imaging is simpler and more straightforward [13]. Due to its no crystal needed, this technique has received broad application in recent years. It is based on Fresnel diffraction theory which can provide an edge-enhancement effect at the boundary between different tissues.

Although phase contrast imaging can produce higher contrast images than conventional methods, these two dimensional images still face a problem of tissue overlap. The sample has to be rotated to a perfect position where we can get a clear view of the interest part. After combining PCI with computed tomography $(\mathrm{CT})$, this problem was worked out [14].

Recent studies demonstrate that PCI has much obvious superiority in soft tissue imaging both on human and small animals such as mice, rats and rabbits [15-17]. This imaging modality exploits refraction variation between different tissues. Therefore, it enables soft tissues imaging, even though they have similar attenuation properties. A number of challenging clinical problems were tested on phase contrast imaging including bone-cartilage imaging, mammography, 
lung airways imaging and liver blood vessel imaging without contrast agent [13].

This paper presents our work during the last three years about medical application of phase contrast imaging, such as hepatic fibrosis imaging, blood vessel visualization and quantitative description of the mouse lung.

\section{MATERIALS AND METHODOLOGY}

\subsection{The Samples}

\subsubsection{Hepatic Fibrosis}

The samples of hepatic fibrosis, induced by human albumin, were prepared in the Liver Research Center, Beijing Friendship Hospital. The mice and rats liver specimens were fixed in $10 \%$ formalin solution. They were cut approximately $2.5 \mathrm{~mm}$ thickness for DEI imaging, 3.5 $\mathrm{mm}$ in diameter and $7 \mathrm{~mm}$ in height for CT.

\subsubsection{Mouse Lung}

It was a 1-day-old mouse, provided by Laboratory Animal Science, Capital Medical University. Before imaging, the mouse was humanely sacrificed by sodium pentobarbital injection overdose. And then it was constrained in a tube, rolled by Kapton film (Dupont, DE, USA), and placed on the sample stage in a vertically up-side-down position.

\subsection{Imaging Methods}

\subsubsection{Diffraction Enhanced Imaging}

Diffraction enhanced imaging (DEI) is an analyzer-based phase contrast imaging method, which is sensitive to refractive effects within the object in addition to the X-ray absorption and scattering by the object [10]. Our experiments were performed at the BL-14B beamline, of Photon Factory, KEK, Japan. Fig. (1) shows the schematic of DEI setup. The white synchrotron beam was first monochromat-

Fig. (1). The schematic of DEI setup and a sample rocking curve.
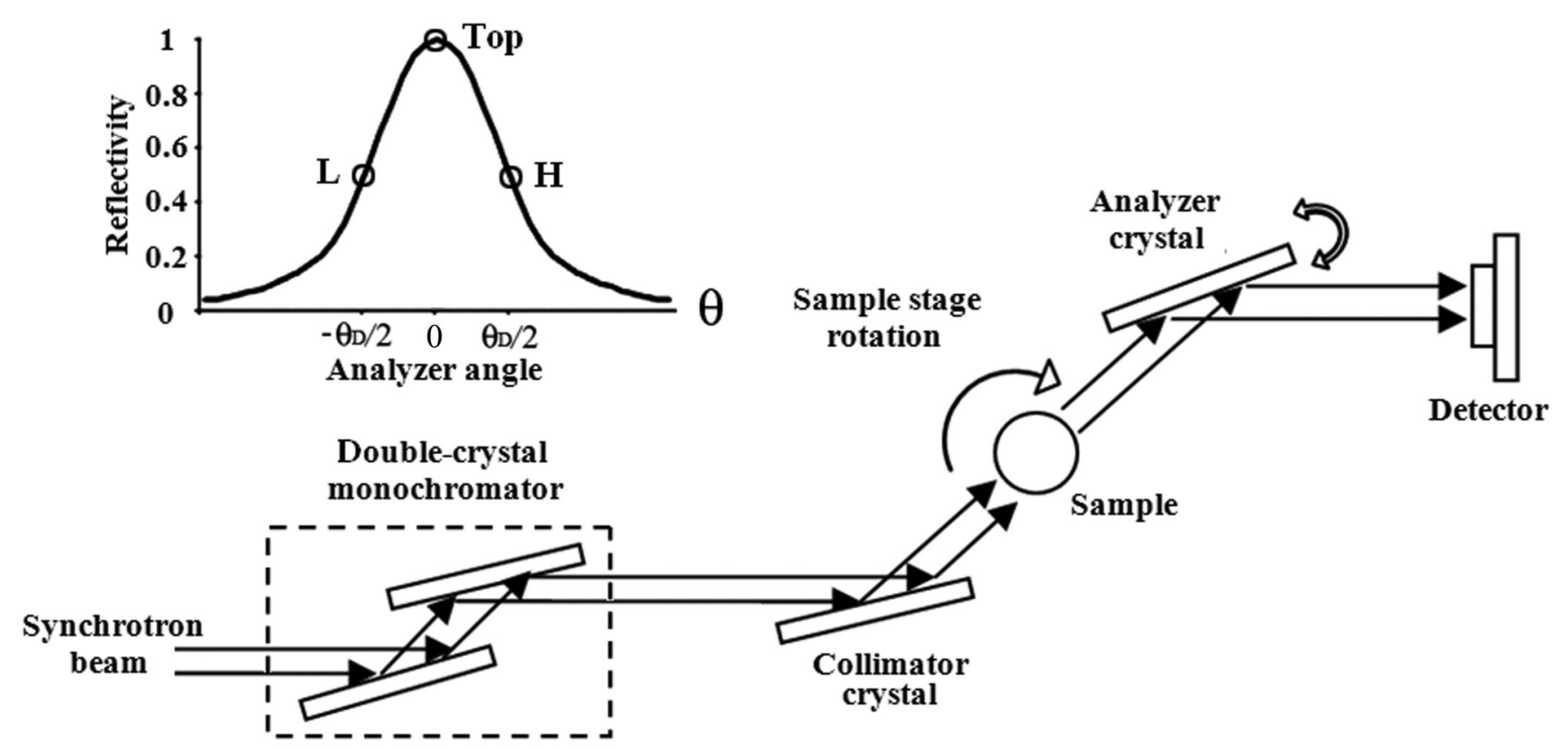

ized by a double-crystal monochromator. The available energy range was between 10 to $60 \mathrm{keV}$. It was adjusted to $15 \mathrm{keV}$ in our experiment. The beams were further collimated by the collimator crystal. The sample placed between the collimator crystal and the analyzer crystal could be rotated on the sample stage. After the sample, the transmitted beams were then deviated from their original direction due to refraction and scattering. According to Brag diffraction theory, the analyzer crystal only reflects photons coming from a particular angle. Finally, the beams reach the detector to obtain an image of the sample. A CCD camera was used as a two-dimensional detector, comprising 1032 pixels $\times 1384$ pixels, with $6.7 \mu \mathrm{m} \times 6.7 \mu \mathrm{m}$ each. In the meridian plane, the analyzer crystal could be rotated within a narrow range, typically several microradians. The relationship between the angle and the reflectivity was characterized by the rocking curve (RC). A sample RC was shown in Fig. (1).

For hepatic fibrosis diagnosis, refraction images were used, which represents refraction angles of the X-ray beams deviated from their original directions. Since refraction images not rely on absorption property, more detailed information of soft tissues can be observed [18]. In order to get pure reflection information, two images $\left(\mathrm{I}_{\mathrm{H}}\right.$ and $\left.\mathrm{I}_{\mathrm{L}}\right)$ were collected when the analyzer is set to full width at half maximum of the rocking curve $\left(\theta= \pm \Delta \theta_{\mathrm{D}} / 2\right)$, with the exposure time of $1 \mathrm{~s}$. The reflection image $\Delta \theta$ can be given by

$$
\Delta \theta=\frac{I_{H} R\left(-\frac{\theta_{D}}{2}\right)-I_{L} R\left(\frac{\theta_{D}}{2}\right)}{I_{L} \frac{d R}{d \theta}\left(\frac{\theta_{D}}{2}\right)-I_{H} \frac{d R}{d \theta}\left(-\frac{\theta_{D}}{2}\right)}
$$

where $\theta_{D}$ is the full width at half maximum of the rocking curve, $R(\theta)$ represents the reflectivity at angle $\theta$.

For CT imaging, the analyzer was set at the peak of the 
rocking curve. Under such circumstance, the image quality could be highly improved by removing most of the scattered beams which are considered as noise in the image. The specimen was rotated around its cylinder axis for $180^{\circ}$ by a step of $1^{\circ}$ with exposure time of $1.5 \mathrm{~s}$.

\subsubsection{In-Line Phase Contrast Imaging}

The principle of IL-XPCI is based on Fresnel diffraction theory. The experiment setup, when using synchrotron radiation light source, is very simple, only requiring a perfect crystal monochromator, a sample stage and a detector. Our experiment was performed at X-ray imaging and biomedical application beamline (BL13W1) of Shanghai Synchrotron Radiation Facility (SSRF). Fig. (2) shows the schematic image of the experiment setup.

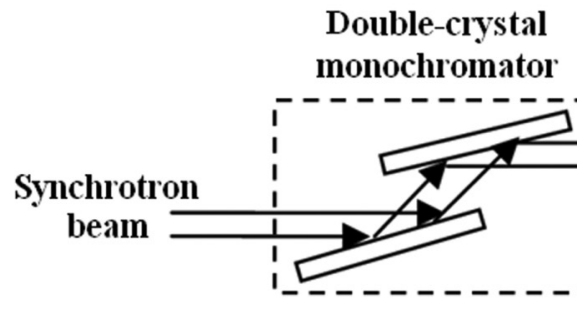

Fig. (2). The schematic of IL-XPCI setup.

SSRF is a third-generation synchrotron facility. Two $\mathrm{Si}(111)$ prefect crystals were used as a monochromator with a tunable energy range from $8 \mathrm{keV}$ to $72.5 \mathrm{keV}$. In our experiment, it was adjusted to $16 \mathrm{keV}$. The highly parallel and monochromatic beam subsequently projected on the object being imaged. When X-ray beams travel through the object, the downstream beams carried the information of absorption and phase shift. After propagating a sufficient distance, in our experiment it was $1.2 \mathrm{~m}$, the phase shifts in the downstream beams were transformed into measurable intensity variations by means of Fresnel diffraction. An Xray sensitive CCD camera, which had maximum $4008 \times 2672$ pixels of $13 \mu \mathrm{m} \times 13 \mu \mathrm{m}$ each, was used as a two-dimensional detector to transform the beam into an image. During the CT data acquisition, the specimen was rotated around its cylinder axis for $180^{\circ}$. The number of projections is 1296 with exposure time of $0.08 \mathrm{~s}$ for each one.

\subsection{Image Processing}

Although phase contrast images have a higher resolution and contrast than conventional images, overlapping of the structures always happen. It is beneficial to obtain threedimensional information of the tissue. By the combination of PCI with computed tomography, three-dimensional observation of the samples is now possible. Cross-sections of the liver and lung were reconstructed by a conventional tomography algorithm - filtered back projection (FBP) [19]. Thus, two-dimensional projection images were transformed into three-dimensional volume datasets. In order to extract blood clot and lung airways of the image, 3D region growing method was used, which is a semi-automatic procedure. The segmentation started from manually choosing a seed point as well as setting an intensity threshold. The method then automatically segmented the $3 \mathrm{D}$ volume datasets by the control of intensity threshold. For the segmentation of vessel walls, threshold-based method was enough to extract them because of their distinct gray scale. Surface rendering method, based on iso-surface detection algorithm, was subsequently performed to give a three-dimensional view of the sample [20]. In many medical fields, such as surgical planning, diagnosis and treatment, surface rendering is a powerful tool to reveal complex spatial relationship of anatomical structures.

\section{MEDICAL APPLICATION RESULTS}

\subsection{Hepatic Fibrosis}

Hepatic fibrosis is a pathological process of connective tissue that results in the architectural derangement of the original collagen structure, altering the structural density, and transforming the underlying molecular order and organization [4]. It can be assessed in six categories, ranging from mild to severe. Recent work suggests that hepatic fibrosis may be cure by treatment at the early stage of the disease [21]. Therefore, distinguishing the degree of liver fibrosis becomes a vital role in successful treatment. Here, DEI method was used to image different stage of liver fibrosis samples. The refraction images of hepatic fibrosis samples from stage one to six were shown in Fig. (3) [22]. They were obtained by taking two images on either side of the rocking curve and calculated using equation (1). After the refraction information extraction, the contours and interfaces of different tissues were highlighted. Different liver fibrosis stages could be distinguished by proliferation or compression on blood vessels and coarseness on liver tissue textures. For example, the liver textures of stage one in Fig. (3) was regularly. Only some small vessels were missing, because they were destroyed at the early disease stage. In stage two, some small vessels destruction parts are pointed by arrows. The arrow in stage five image indicates blood flow ways reconstruction. Stage six is cirrhosis; the apparent septum is pointed by arrow. These analyses were confirmed by histological pictures of the corresponding samples shown in Fig. (3). The histological assessment was done by a pathological specialist. The further image texture analysis we did also supported these results.

\subsection{Microvessels Visualization}

Microvascular disease is being increasingly recognized as an important risk factor in the setting of hypertension, diabetes, coronary heart disease (CHD), cancer, etc. [2, 23, 24]. Current imaging methods were hampered by the spatial resolution and contrast when imaging microvessels. Thanks to the breakthroughs in phase contrast imaging, non-invasive 


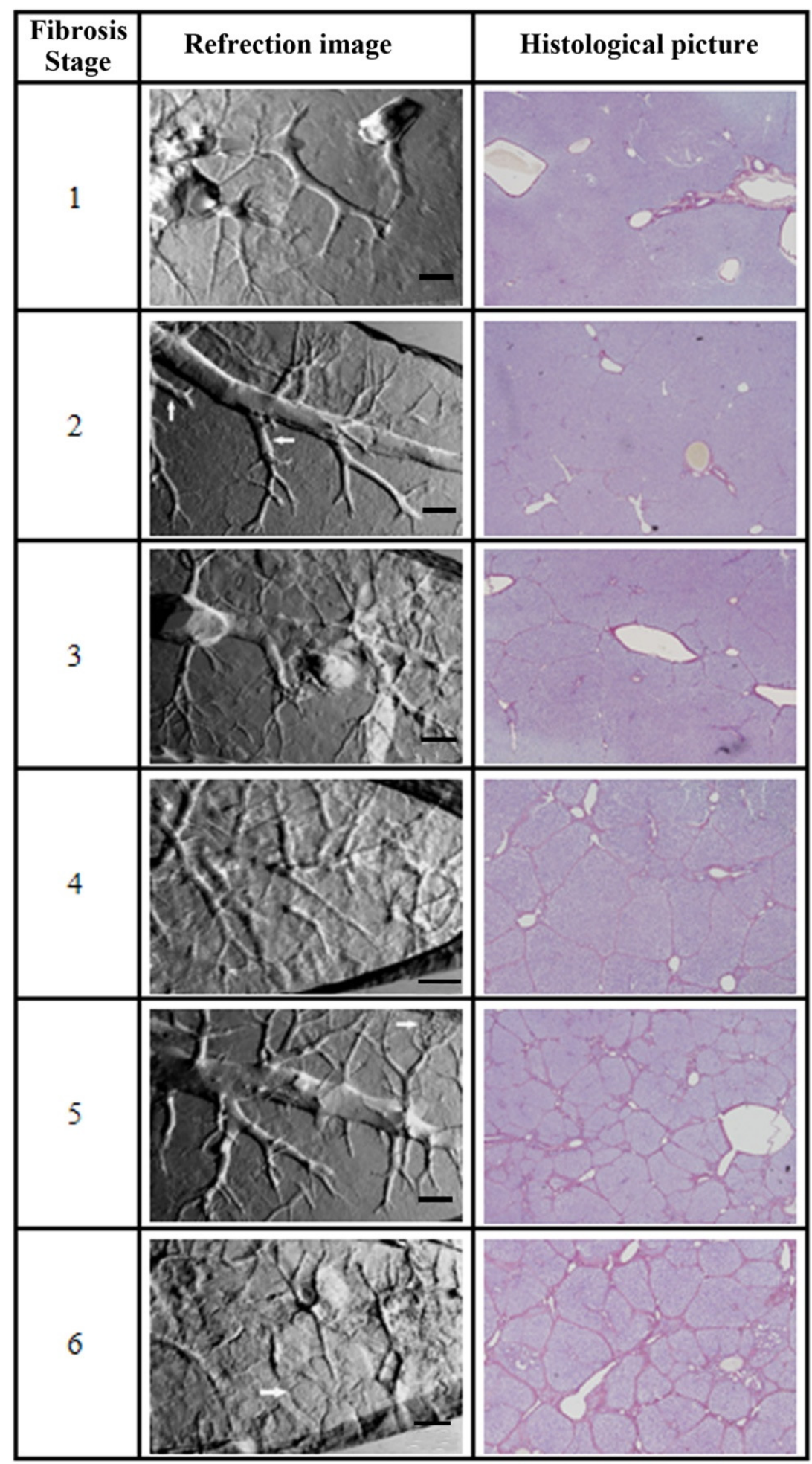

Fig. (3). The refraction images of hepatic fibrosis samples and the corresponding histological images from stage one to six. The bars in the refraction images are $500 \mu \mathrm{m}$.

visualization of the microvessels without contrast agent is now possible. Fig. (4) shows the three-dimensional reconstruction results of a section of mouse liver blood vessel with the diameter of about $300 \mu \mathrm{m}$ [25]. Different materials were indicated by their different colors. By region growing, the blood clot inside the vessel was segmented. It is in blue color. The inner layer and the outer layer of the vessel wall are in green and red color respectively. The 3D model can be cut at any angle. As shown in Fig. (4b), a plane was used to cut the vessel in the direction perpendicular to the vessel axis. The cross section was also displayed in Fig. (4b). In this regard, the stenosis rate of the vessel can be quantitatively measured which could yield criteria for assessing stenosis. 

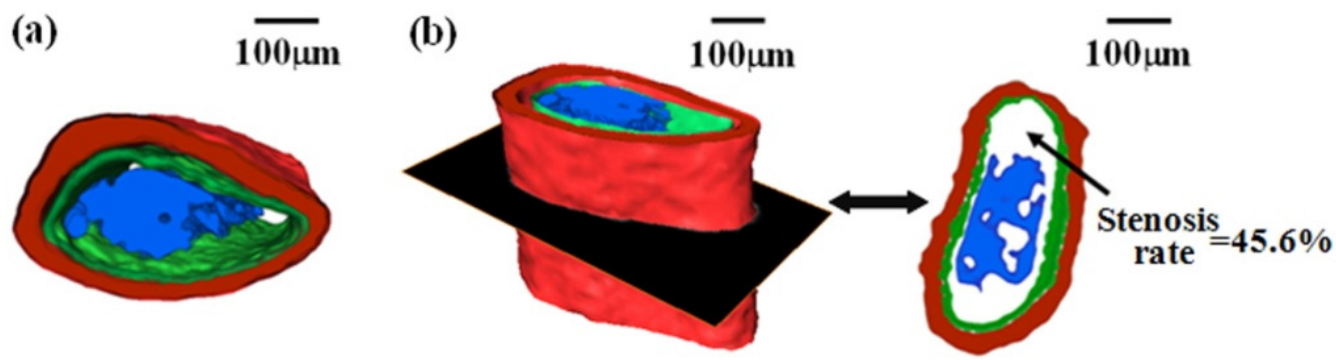

Fig. (4). One segment of the blood vessel. (a) top view (b) one profile view and the cross-section image.

\subsection{Lung}

For conventional lung imaging, it could hardly display any microstructures of the sample without contrast agent. The lung is a respiration organ composed of sponge-like soft tissues. $90 \%$ of the lung is filled with air. At the interface of air and tissue, the refractive index changes greatly. In phase contrast imaging, this will lead to a remarkable edge enhancement of the airways, even though they have similar absorption properties [26]. As shown in Fig. (5a), the structures in mouse thorax such as bronchi, bronchioles, and alveoli can be clearly discerned from the CT reconstruction slice. Surface rendering method was used to give a 3D view of the whole airway including alveoli (Fig. 5b) [27]. This 3D model can be cut at any angle, varied in size, and rotated in real time. The alveoli are essential as the final branching of the respiratory through which gas exchange occurs. For the investigation of inhaled medicines' function and mechanism, the visualization of the subtle airway is of considerable value. There are also potential uses in respiratory disease diagnosis when the morphology and distribution of alveoli were modified such as pneumonia, asthma, chronic bronchitis and emphysema. Fig. (5c) is a detailed demonstration of the terminal branching of the lung. These images provided a magnified view of the alveoli. The diameter and surface area of the alveoli were measured.

\section{DISCUSSION}

So far histological biopsy is still the gold standard in hepatic fibrosis assessment. However, the risk of morbidity and even mortality cannot be avoided. Taking this into consideration, non-invasive method for liver fibrosis assessment is preferable. Nevertheless, the currently used imaging techniques such as ultrasound, X-ray CT and MR cannot detect hepatic fibrosis by now [28]. In our experiment, DEI technique showed good ability to
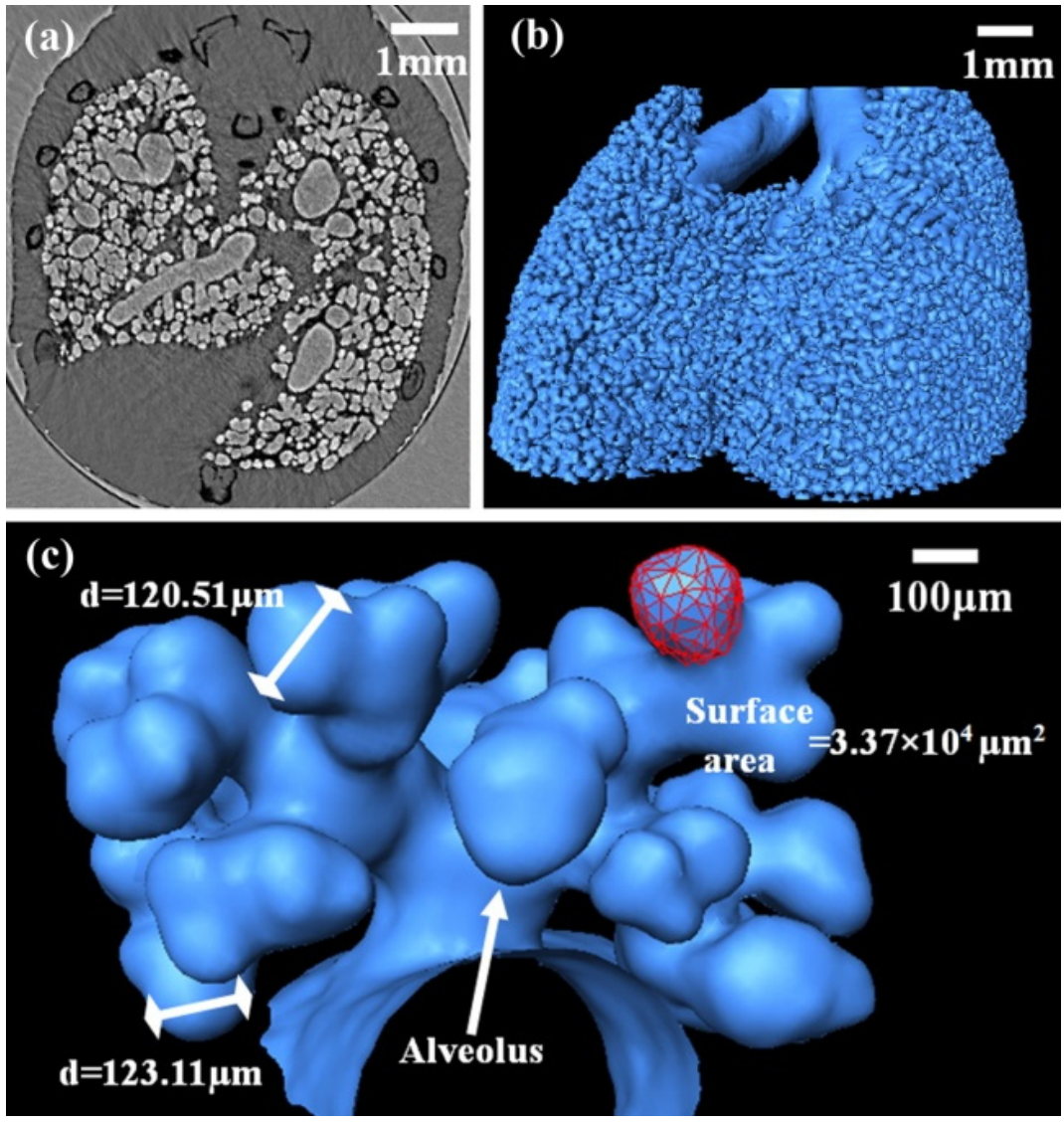

Fig. (5). Images of the lung. (a) one CT slice (b) the bronchi and alveoli (c) a magnified view of the terminal branching of the lung. 
distinguish hepatic fibrosis stages. But this is still in the experimental stage. More hepatic fibrosis samples should be evaluated to examine the possibility and accuracy of this technique. Meanwhile we had to face a problem of superimposition of the structures inside the tissue. Therefore, $3 \mathrm{D}$ visualization method was used in our subsequent study on blood vessels in liver.

Visualization of microvessels is real challenging, especially for non-invasive imaging. Existing imaging methods focused exclusively on the detection of vessel exterior. In most cases, contrast agent had to be used. Although contrast agent is generally safe, bad side effects do sometimes occur [29]. Selective coronary angiography is the most commonly used method for coronary artery imaging. It has complex operation associated with a small but definite risk of complications. A non-invasive and high-resolution vascular imaging method would be of clinical benefit. In our study, we demonstrated that DEI can produce enough contrast when observing blood clot in the vessel. The 3D reconstruction results accurately depicted the complex vascular structures in addition to the morphology of the blood clot inside the vessel. Conventional imaging methods have a limited capability to non-invasively visualize the structures inside the vessels, especially in three-dimension. While many clinical findings show that coronary vessel with a major stenosis is a leading cause of most myocardial infarctions; the size of a single embolus might determine its clinical consequences [30]. The 3D blood vessel model we presented in this paper can be used to perform vessel stenosis study which could yield criteria for assessing stenosis. It allowed for the detection of thrombus formation and progression which could reduce the risk of heart attack and other disease with thrombus.

A number of researchers have imaged lungs using phase contrast imaging. Lungs of mice, rats and rabbits were imaged both by IL-XPCI and DEI $[13,16]$. Though the lung were highly visible by the use of PCI, due to the large number of alveoli minor structures were averaged out in two-dimensional images [17]. For three-dimensional lung visualization, many attempts had been made. The 3D model of the lung airways had been generated, but the alveoli were not included [31]. During our study, we have successfully generated the 3D model of the mouse lung. Even at the final branching of the respiratory tree, the alveoli are perfectly displayed. Furthermore, we measured the diameter of two alveoli and calculated the surface area of one alveolus, which are crucial for better understanding of the alveoli morphology. Moreover, this may play a positive role for physicians in providing more precise diagnosis related to alveolar size such as chronic bronchitis, emphysema and drowning, and subsequently giving better treatment. It is known that lung cancer is usually derived from epithelial cells and near a central bronchus. For early lung cancer screening, conventional X-ray CT and MRI are not available [5]. Phase contrast imaging of lung offers the hope that early stage lung cancer will be detected by the edge-enhancement effect of the airway associated with high imaging resolution. However, more studies must be done to come to the conclusion that PCI is advantageous in terms of cancer detection.
The limitation of our study was that live animals were not imaged. Phase contrast imaging is a high resolution imaging technique; the motion artifacts of breathing and heart beat will lead to serious image blur when reconstructing CT slices. Possible solution is to substantially reduce the imaging time and finish a $\mathrm{CT}$ in one respiration phase.

In this paper, we proved the ability of PCI in hepatic fibrosis and lung imaging. The microstructures like the blood clot inside the microvessel and subtle alveoli were reconstructed in three dimension. We are currently working on the imaging of mouse with lung cancer to confirm our hypothesis of lung cancer detection using PCI. At the same time, myocardial infarction samples are added for the study of coronary microembolization. More pathological samples will be tested in our PCI experiments. We hope this highresolution and non-invasive imaging technique can be used in disease diagnosis in the near future, and now it is in progress.

\section{CONCLUSION}

In summary, this paper describes the medical applications of phase contrast imaging on the study of hepatic fibrosis, microvessel visualization and quantitative description of the mouse lung. DEI technique facilitates the non-invasive imaging of the hepatic fibrosis in $2 \mathrm{D}$, which provides a straightforward way of hepatic fibrosis diagnosis. The further computed tomography experiment realized the detection and visualization of microvessel in 3D. The blood clot inside the vessel was obtained by means of image processing. Moreover, the mouse lung has been investigated by IL-XPCI. 3D images of the whole lung airways have been acquired. The single alveolus has been visualized in 3D and assessed quantitatively, resulting in a comprehensive description of the lung microstructures. All the results showed that phase contrast imaging has the ability to offer enormous benefit for the soft tissue imaging. Although a great deal of work should be done to apply this technique into clinical practice.

\section{ACKNOWLEDGMENTS}

The authors acknowledge the staffs from beamline BL14B of the Photon Factory in Tsukuba, Japan and BL13W1 of Shanghai Synchrotron Radiation Facility (SSRF) for their kindly support for the experiments. The research was supported by the Natural Science Foundation of China, Grant Nos. 60532090 and 30770593.

\section{CONFLICT OF INTEREST}

None declared.

\section{REFERENCES}

[1] Jacob RE, Carson JP, Gideon KM, Amidan BG, Smith CL, Lee KM. Comparison of two quantitative methods of discerning airspace enlargement in smoke-exposed mice. PLoS ONE 2009; 4(8): e6670.

[2] Dall'Oglio MF, Ribeiro-Filho LA, Antunes AA, et al. Microvascular tumor invasion, tumor size and Fuhrman grade: a pathological triad for prognostic evaluation of renal cell carcinoma. J Urol 2007; 178(2): 425-8.

[3] Reitz C, Mayeux R. Endophenotypes in normal brain morphology and alzheimer's disease: a review. Neuroscience 2009; 164(1): 17490. 
[4] Standish RA, Cholongitas E, Dhillon A, Burroughs AK and Dhillon AP. An appraisal of the histopathological assessment of liver fibrosis. Gut 2006; 55(4): 569-78.

[5] Biederer J, Both M, Graessner J, et al. Lung morphology: fast MR imaging assessment with a volumetric interpolated breath-hold technique: initial experience with patients. Radiology 2003; 226(1): 242-49.

[6] Hoffman PC, Mauer AM, Vokes EE. Lung cancer. Lancet 2000; 355(9202): 479-85.

[7] Wilkins SW, Gureyev TE, Gao D, Pogany A, Stevenson AW. Phase-contrast imaging using polychromatic hard X-rays. Nature 1996; 384(6607): 335-8

[8] Wu X, Liu H. Clinical implementation of phase contrast X-ray imaging: theoretical foundation and design considerations. Med Phys 2003; 30(8): 2169-79.

[9] Momose A, Takeda T, Itai Y, Hirano K. Phase-contrast X-ray computed tomography for observing biological soft tissues. Nat Med 1996; 2(4): 473-5.

[10] Davis T J, Gao D, Gureyev T E, Stevenson AW, Wilkins SW. Phase-contrast imaging of weakly absorbing materials using hard X-rays. Nature 1995; 373(6515): 595-8.

[11] Pfeiffer F, Weitkamp T, Bunk O, David C. Phase retrieval and differential phase-contrast imaging with low-brilliance X-ray sourced. Nat Phys 2006; 2(4): 258-61.

[12] Lewis RA, Hall CJ, Hufton AP, et al. X-ray refraction effects: application to the imaging of biological tissues. Br J Radiol 2003; 76 (905): 301-8

[13] Lewis RA. Medical phase contrast $x$-ray imaging: current status and future prospects. Phys Med Biol 2004; 49(16): 3573-83.

[14] Takeda T, Momose A, Hirano K, Haraoka S, Watanabe T, Itai Y. Human carcinoma: early experience with phase-contrast X-ray CT with synchrotron radiation-comparative specimen study with optical microscopy. Radiology 2000; 214(1): 298-301.

[15] Ohtsuka S, Hyodo K, Jin W, et al. Overview of clinical intravenous coronary angiography both in Japan and at ESRF. Nucl Instrum Meth A 2005; 548(1-2): 78-83.

[16] Kitchen MJ, Lewis RA, Yagi N, et al. Phase contrast X-ray imaging of mice and rabbit lungs: a comparative study. Brit $\mathrm{J}$ Radiol 2005; 78(935): 1018-27.

[17] Suzuki Y, Yagi N, Uesugi K. X-ray refraction-enhanced imaging and a method for phase retrieval for a simple object. J Synchrotron Radiat 2002; 9: 160-5.
[18] Chapman D, Thomlinson W, Johnston RE, et al. Diffraction enhanced X-ray imaging. Phys Med Biol 1997; 42(11): 2015-25.

[19] Kak AC, Slaney M Principles of computerized tomographic imaging. New York: IEEE Press 1988.

[20] Luo S and Zhou G. Medical Image Processing and Analysis, 2st ed. Beijing: Science Press; 2010 [in Chinese]

[21] Dienstag JL, Goldin RD, Heathcote EJ, et al. Histological outcome during long-term lamivudine therapy. Gastroenterology 2003; 124(1): 105-17.

[22] Li H, Zhang L, Wang XY, et al. Investigation of hepatic fibrosis in rats with X-ray diffraction enhanced imaging. Appl Phys Lett 2009, 94(12): 124101-3.

[23] Zhu XY, Daghini E, Chade AR, et al. Simvastatin prevents coronary microvascular remodeling in renovascular hypertensive pigs. J Am Soc Nephrol 2007; 18(4): 1209-17.

[24] Gomes MB, Matheus AS, Tibiriçá E. Evaluation of microvascular endothelial function in patients with type 1 diabetes using laserDoppler perfusion monitoring: Which method to choose? Microvasc Res 2008; 76(2): 132-3.

[25] Zhang $\mathrm{L}$, $\mathrm{Hu} \mathrm{C}$, Zhao $\mathrm{T}$, Luo $\mathrm{S}$. Noninvasive visualization of microvessels using diffraction enhanced imaging. Eur J Radiol 2010 [Epub ahead of print].

[26] Hausmann R, Bock H, Biermann T, Betz P. Influence of lung fixation technique on the state of alveolar expansion-a histomorphometrical study. Legal Med 2004; 6(1): 61-5.

[27] Zhang L, Li D, Luo S. Non-invasive microstructure and morphology investigation of the mouse lung: qualitative description and quantitative measurement. PLoS ONE 2011;6(2): e17400.

[28] Castera L, Forns X, Alberti A. Non-invasive evaluation of liver fibrosis using transient elastography. J Hepatol 2008; 48(5): 83547.

[29] Cutroneo P, Polimeni G, Curcuruto R, Calapai G, Caputi AP. Adverse reactions to contrast media: An analysis from spontaneous reporting data. Pharmacol Res 2007; 56(1): 35-41.

[30] Bahrmann P, Werner GS, Heusch G, et al. Detection of coronary microembolization by doppler ultrasound in patients with stable angina pectoris undergoing elective percutaneous coronary interventions. Circulation 2007; 115(5): 600-8.

[31] Parsons DW, Morgan K, Donnelley M, et al. High resolution visualization of airspace structures in intact mice via synchrotron phase-contrast X-ray imaging (PCXI). J Anat 2008; 213(2): 217 27.

(C) Zhang and Shuqian; Licensee Bentham Open.

This is an open access article licensed under the terms of the Creative Commons Attribution Non-Commercial License (http://creativecommons.org/licenses/by-nc /3.0/) which permits unrestricted, non-commercial use, distribution and reproduction in any medium, provided the work is properly cited. 